\title{
Photocatalytic degradation of methylene blue by composite TiO2-diatomite sheets under UV irradiation
}

\author{
Jingzheng,Weng ${ }^{1,2, a}$, Feng Gao ${ }^{2}$,Lihui, Chen ${ }^{1}$, Renhui,Qiư ${ }^{3, \text { b, }}$ \\ ${ }^{1}$ College of Material Engineering,Fujian Agriculture and Forestry University, Fuzhou, Fujian Province \\ 350002, China \\ ${ }^{2}$ College of Materials Science and Engineering ,Fujian Normal University ,Fuzhou, Fujian Province \\ 350007,China \\ ${ }^{3}$ College of Transportation and Civil Engineering, Fujian Agriculture and Forestry University, \\ Jinshan, Fuzhou, Fujian Province 350002, P. R. China \\ a jackyweng@vip.163.com, ${ }^{b}$ renhuiqiu@fafu.edu.cn * corresponding author
}

\begin{abstract}
Keywords: Photocatalytic degradation; methylene blue; $\mathrm{TiO}_{2}$
Abstract. Titanium dioxide $\left(\mathrm{TiO}_{2}\right)$ photocatalyst and diatomite adsorbent were made into a paper-like composite by a papermaking technique using pulp and ceramic fibers as sheet matrix. Simulated wastewaters that contain methylene blue (MB) bleached in photocatalytic $\mathrm{TiO}_{2}$-diatomite sheet was investigated under UV irradiation. The $\mathrm{TiO}_{2}$-diatomite sheet could remove the $\mathrm{MB}$ from water completely under UV irradiation. The characteristics of composite sheets were studied by XRD and SEM techniques. It was found that the composite $\mathrm{TiO}_{2}$-dioatomite composite sheet could be utilized semi-permanently and was easier to handle than the original $\mathrm{TiO}_{2}$ powders in aqueous media. Consequently, a composite $\mathrm{TiO}_{2}$-diatomite sheet can be utilized as a high functional material to photodegradate organic pollution.
\end{abstract}

\section{Introduction}

Textile dyes and other commercial dyestuffs such as azo-dye have become a focus of environmental remediation efforts in the last few years[1,2] . Dye factories release a significant quantity of wastewaters containing dyes and other organic species. The textile and photographic industries also dispose large volumes of similar wastewaters that cause significant environmental pollution. More than $1.6 \times 109 \mathrm{~m}^{3}$ dye containing effluents in China are discharged annually[3]. Water remediation may involve physical, chemical, and biological treatments. Most wastewater industrial treatment processes have limitations that restrict their applications.

Dyes are insufficiently removed by conventional sewage plant treatment because of their high biochemical stability, their relatively high molecular weight, and high water solubility relative to their excellent all-round fastness. Considerable attention has been expended recently on the photocatalytic oxidative degradation of these wastewaters. In particular, the photocatalytic oxidation of organic pollutants by titanium dioxide $\left(\mathrm{TiO}_{2}\right)$ has attracted much attention as a promising chemical procedure for environmental cleanup. $\mathrm{TiO}_{2}$ is nontoxic, inexpensive, and photoactivated by radiation energy in the near-UV range. Decomposition of organic contaminants is mediated by a series of radical reactions initiated by the active oxygen species formed on the surfaces of the $\mathrm{TiO}_{2}$ crystal. $\mathrm{Thus} \mathrm{TiO}_{2}$ photocatalysis is effective for the degradation of a wide range of organic pollutants in water; however, the small particle size of the powder means that a microfiltration stage is necessary for catalyst recovery. Moreover, the inorganic powders frequently cause secondary pollutions. Therefore, much research has been devoted to immobilizing $\mathrm{TiO}_{2}$ and other powder materials onto a suitable supporting matrix for practical applications. Many papers have reported that $\mathrm{TiO}_{2}$ coating with substances such as activated carbon[4,5], zeolite[6,7,8],glass[9,10,11], etc.

In the present work, the inorganic powders of $\mathrm{TiO}_{2}$ and diatomite were made into a flexibe, paper-like product by the papermaking technique. The composite sheets were applied to decompose $\mathrm{MB}$ in an aqueous solution under UV irradiation. The efficiency of MB degradation by the composite 
$\mathrm{TiO}_{2}$-diatomite sheets was investigated for various ratios of $\mathrm{TiO}_{2}$ to diatomite through the quantitative determination in the composite sheets.

\section{Experimental}

\section{Materials}

Phenol $\left(\mathrm{C}_{6} \mathrm{H}_{5} \mathrm{OH}\right.$, Sigma-Aldrich, $99 \%$ ), $\mathrm{TiO}_{2}$ powder ( Tianjin Fucheng Chemical Reagents Factory, Titanium Dioxide), diatomite (Guangdong Geology and Mineral Products), eucalypt pulp slurry ( $35^{\circ} \mathrm{SR}$ ) were purchased commercially and used as received. Two types of flocculants, polydiallyl dimethylammonium chloride (PDADMAC; molecular weight $(\mathrm{Mw})$, ca. $8 \times 10^{4}$; Sigma-Aldrich) and anionic polyacrylamide (A-PAM, HH-351, Mw, ca. $2.25 \times 10^{6}$, self-prepared) were used as retention aids in a dual polyelectrolyte system. The phenol and other chemicals ranked as reagent grade in purity and were used without further purification.

\section{Preparation of composite sheets}

Handmade paper sheets with a pulp grammage of $110 \mathrm{~g} / \mathrm{m} 2$ were prepared according to TAPPI Test Methods T205 by using ZQJ2-B papermaking machine. Pulp slurry was mixed with PDADMAC ( $0.3 \mathrm{wt} \%$ of the dry pulp weight), followed by the sequential addition of inorganic suspensions which contain $\mathrm{TiO}_{2}$, diatomite, and A-PAM ( $0.9 \mathrm{wt} \%$ of the dry pulp weight). Then the mixture was pressed at $500 \mathrm{KPa}$ for $5 \mathrm{~min}$. Afterward, the wet sheets were oven-dried at $105^{\circ} \mathrm{C}$ for $30 \mathrm{~min}$. The composite sheets contain three components, i.e., $\mathrm{TiO}_{2}(0 \sim 35 \mathrm{wt} \%)$, diatomite $(35 \sim 0 \mathrm{wt} \%)$, and pulp $(65 \mathrm{wt} \%)$. Four types of composite sheets were prepared with different weight ratios of $\mathrm{TiO}_{2}$ to diatomite, i.e., 2:1, 1:1, 1:2 and 1:4 in terms of weight. The weight of dry sheets was measured by analytical electron balance and their retention ratios were calculated as the dry weight after applying in solution divided by the original dry weight.

Retention ratios of diatomite, pulp and $\mathrm{TiO} 2$ after application of the sheets in aqueous solution were calculated and the results were shown in table1. The paper sheets obtained were conditioned for more than $24 \mathrm{~h}$ at $23{ }^{\circ} \mathrm{C}$ with a relative humidity of $50 \%$ before using in experiments.

\section{UV photodegradation and analyses}

The MB solution (various concentration, $25 \mathrm{~mL}$ ) prepared with deionized water was poured into a glass vessel, which did not exhibit near-UV absorption. Either a composite sheet(area:39.25 cm2) was put into the reaction vessel which was irradiated with an UV $\operatorname{lamp}(20 \mathrm{~W}, \lambda=254 \mathrm{~nm})$. The sheet of illumination area was about $39.25 \mathrm{~cm}^{2}$ and $15 \mathrm{~cm}$ distance from UV lamp. The adsorption and photocatalytic decomposition of MB were studied at room temperature. Analytical samples were taken from the solution at the given time intervals,.

The concentration of MB concentration was analyzed by a UV-visible spectrophotometer at the wavelength of $664 \mathrm{~nm}$ and the decolouring efficiency of MB solution was calculated as follows:

$$
T=\frac{A_{0}-A}{A_{0}} * 100 \%
$$

$\mathrm{T}$ : removal ratio of the MB; $\mathrm{A}_{0}$ : initial absorbency of MB solution of; A: analytical absorbency of sample solution.

After this experiment, the paper sheet removed from the solution and dried in an oven at $105^{\circ} \mathrm{C}$ for $30 \mathrm{~min}$. The sheet was repeatedly utilized in the same experiment.

\section{Characterization of sheets' surface and crystalization}

The crystalline phase of the sheet was analyzed by X-ray powder diffraction (XRD) using a MiniFlex 600 diffractometer (Rikagu, Japan) with $\mathrm{Cu}$ Ka radiation (wavelength $=1.54 \mathrm{~A}^{\circ}$ ). Diffractograms of powders were recorded in $2 \mathrm{~h}$ configuration from 10 to $90^{\circ}$ with an increment of $0.02^{\circ}$. 


\section{Results and discussion}

\section{Effect of $\mathrm{TiO}_{2}$-diatomite weight ratio in the composite sheets on Physical characteristics}

Table. 1 gives the data for the sheet samples used in the experiment. Retention of materials including diatomite, ceramic fiber, pulp and $\mathrm{TiO}_{2}$ was calculated. These values were corrected for the additive materials themselves.

Table.1 Physical characteristics of composite sheets

\begin{tabular}{|c|c|c|c|c|c|}
\hline The weight ratios of $\mathrm{TiO}_{2}$ to diatomite & $2: 1$ & $1: 1$ & $1: 2$ & $1: 4$ & $1: 8$ \\
\hline Retention ratio $(\%)$ & 94.4 & 96.9 & 96.1 & 96.6 & 96.0 \\
\hline Rupture length $(\mathrm{km})$ & 0.265 & 0.393 & 0.413 & 0.376 & $1^{0.37}$ \\
\hline Tensile strength $(\mathrm{kN} / \mathrm{m})$ & 0.551 & 0.711 & 0.814 & 0.859 & $2^{0.93}$ \\
\hline
\end{tabular}

It shows the weight ratios of $\mathrm{TiO}_{2}$ and diatomite were little effect on the retention of the raw materials. The well Rupture length of the composite $\mathrm{TiO}_{2}$-diatomite sheets with $\mathrm{TiO}_{2}$ dioatomite ratio of $1: 2$ was about $0.413 \mathrm{~km}$. The composite $\mathrm{TiO}_{2}$-diatomite sheet showed a little tendency to increase tensile strength with increasing $\mathrm{TiO}_{2} /$ diatomite ratio in the sheet.

\section{The Influence of initial MB solution's concentration on the photocatalytic reaction by the composite $\mathrm{TiO}_{2}$-diatomite sheet}

Fig.1 shows photodecomposition behavior of differently initial MB solution's concentration by composite sheets with $\mathrm{TiO}_{2}$ /diatomite ratio of 1:4. At the given time at 120 minutes, analytical samples were taken from the solution. While the initial concentration of MB solution was $10 \mathrm{mg} / \mathrm{L}$, the highest photodecomposition behavior was shown. It was thought that with increasing of the concentration, a number of UV photons decreases as there were assimilated by MB molecule. However, electrons was excited by the energy (such as ultraviolet light). Because the intensity of the UV light was stable, the number of electrons that were promoted also was limited. Therefore, the degradation efficiency of composite sheet may depend upon the experiment conditions such as the pollutant concentration and light intensity.

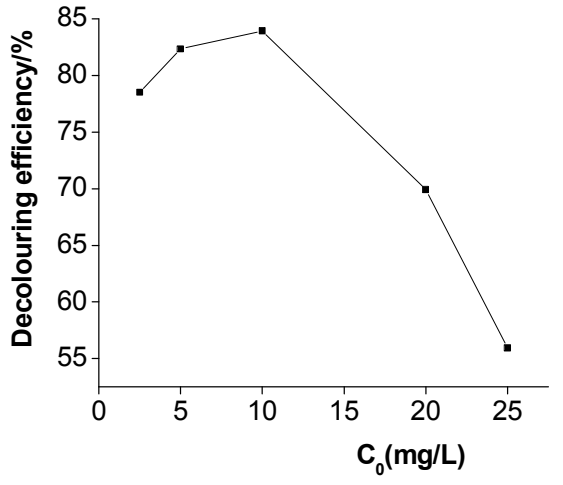

Fig.1. Photodecomposition behavior of differently initial concentration $\mathrm{MB}$ solution by composite sheet: $\mathrm{TiO}_{2}$ : diatomite $=1: 4, \mathrm{UV}$ illumination: $2.0 \mathrm{~h}$

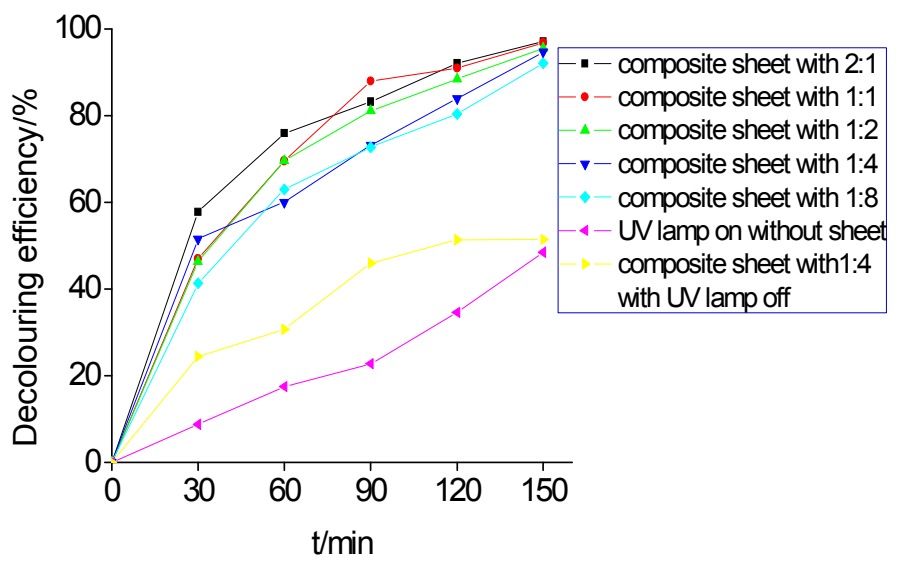

Fig.2..Decolouring efficiency of MB solution in contrast with composite $\mathrm{TiO}_{2}$-diatomite sheets irradiated with UV. 


\section{Effect of the different $\mathrm{TiO}_{2}$ /diatomite ratio in the composite sheets}

Fig.2 indicates the effect of the different $\mathrm{TiO}_{2} /$ diatomite ratio in the composite sheets in $\mathrm{MB}$ concentration $\left(\mathrm{C}_{0}=10 \mathrm{mg} / \mathrm{L}\right)$ under $\mathrm{UV}$ irradiation in order to get the optimum ratios of $\mathrm{TiO}_{2} /$ diatomite in the composite sheets. The initial rate of decolouring efficiency by the composite sheet showed a little improvement with more content of $\mathrm{TiO}_{2}$ in the sheet. After 150 minutes, the removal efficiency of all the samples had little difference and the MB could be removed efficiently by all samples.

And two compared experiments without sheet or without UV irradiation showed obvious different results. Thought the concentration of $\mathrm{MB}$ was also decreased, but the MB could not be removed completely.

\section{Permanency of TiO2-diatomite composite sheet}

In order to assess whether a composite sheet could repeatedly removed the environmental pollutants, repeat tests were carried out. After each photodegradation reaction, the composite sheet removed from the solution and dried in an oven at $105{ }^{\circ} \mathrm{C}$ for $30 \mathrm{~min}$. Then the same sample sheets were repeatedly utilized, Analytical samples were taken from the solution at the given time 150 minutes. The results are shown in Fig.3. It shows the MB could be efficiently removed by the composite sheet and the losing quality ratio of sheet after ten times repeatedly utilized was less $5 \%$ in contrast with original paper sheet. Few powders dispersed from the composite sheet in water result in the weight losing. These results indicate that the composite sheets can continuously remove environmental pollutants and can be utilized semi-permanently.

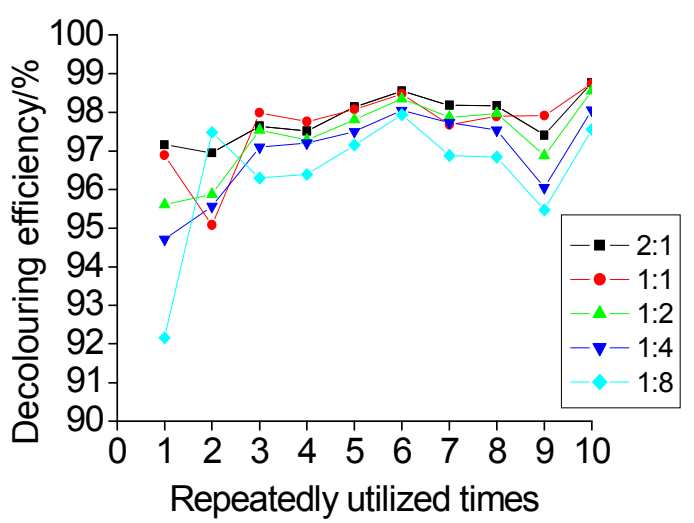

Fig.3 Decolouring efficiency of $\mathrm{MB}$ solution in contrast with repeated irradiation of composite $\mathrm{TiO}_{2}$-diatomite sheets under UV irradiation. TiO2: diatomite $=1: 4, \mathrm{UV}$ illumination: $2.5 \mathrm{~h}$

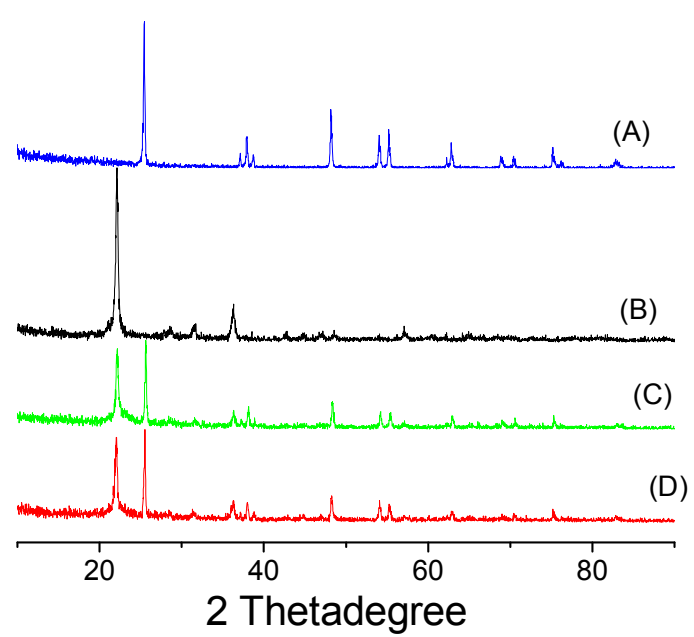

Fig. 4 XRD patterns of samples; (A): $\mathrm{TiO}_{2}$ powder; (B): diatomite powder; (C): $\mathrm{TiO}_{2} /$ diatomite (1:2) composite sheet before photocatalytic utilization; (D): $\mathrm{TiO}_{2} /$ diatomite (1:2) Composite sheet after ten times photocatalytic utilization

\section{The XRD patterns}

Fig.4..shows the X-ray diffraction patterns of some select samples. The diffraction angle degree of $\mathrm{TiO}_{2}$ powder was $25.46^{\circ}, 38.72^{\circ}$, and $48.18^{\circ}$, while that of diatomite powder was $22.10^{\circ}$ and $36.32^{\circ}$. And above diffraction angles all existed in the X-ray diffraction patterns of the $\mathrm{TiO}_{2}$-diatomite composite sheet. It can conform the $\mathrm{TiO}_{2}$ powder and diatomite powder have been immobilized in the sheet, and their crystal structure have nochange. 
The X-ray diffraction patterns of the $\mathrm{TiO}_{2}$-diatomite sheet after photocatalytic utilization was similar to that of the $\mathrm{TiO}_{2}$-diatomite sheet before photocatalytic utilization. It can proved that the crystal structure of $\mathrm{TiO}_{2}$-diatomite composite sheet had little change after ten times photocatalytic utilization.

\section{Conclusions}

A composite $\mathrm{TiO}_{2}$-diatomite sheet was very effective for the photocatalytic decomposition of $\mathrm{MB}$ and had enough intension, it can continuously resmove environmental pollutants and can be utilized semi-permanently. The degradation efficiency of composite sheet may depend upon the experiment conditions such as the pollutant concentration and light intensity. These results lead to the conclusion that the composite $\mathrm{TiO}_{2}$-diatomite sheet is potentially applicable as a high functional and new material, which can completely remove toxic substances from the environment and has the characteristics of being easy to handle and flexible in processing. Further details on the application for composite $\mathrm{TiO}_{2}$-diatomite sheet will be reported in the following articles.

\section{Acknowledgment}

This financial support of thiswork was from Fujian Province Education Department Technological Project under project number of JB12023.

\section{References}

[1] R. Ganesh, G.D. Boardman, D. Mochelson, Water Res. 28 (1994) 1367.

[2] E.J. Weber, R.L. Adams, Environ. Sci. Technol. 29 (1995) 113.

[3] F. Zhang, J. Zhao, T. Shen, H. Hidaka, E. Pelizzetti, N. Serpone, Appl. Catal. B.15 (1998) 147

[4] B. Tryba, A.W. Morawski, M. Inagaki. Applied Catalysis B: Environmental.41 (2003) 427-433

[5] Amjad H. El-Sheikh, Alan P. Newman, Hafid Al-Daffaee, Suki Phull, Neil Cresswell, Steven York. Surface \& Coatings Technology. 187 (2004) 284-292

[6] Jiang Yinsan, Jin Weiqun,Zhang Jun,Fang Songsheng.Journal of Inorganic Materials.2002,17(6): $1031-1035$

[7] H. ICHIURA, T. KITAOKA, H. TANAKA. JOURNAL OF MATERIALS SCIENCE.37 (2002) $2937-2941$

[8] S. Easwaramoorthi, P. Natarajan. Microporous and Mesoporous Materials. 86 (2005) 185-190

[9] S. Horikoshi, N. Watanabe, H. Onishi, H. Hidaka, N. Serpone. Applied Catalysis B: Environmental.37(2002) 117-129

[10] Kuo-Hua Wang, Hung-Huan Tsai, Yung-Hsu Hsieh. Applied Catalysis B: Environmental. 17 (1998) 313-320

[11] Elina Portjanskaja, Marina Krichevskaya, Sergei Preis, Juha Kallas. Environ Chem Lett. (2004) $2: 123-127$ 